\title{
Coherence loss in phase-referenced VLBI observations
}

\author{
I. Martí-Vidal ${ }^{1, \star}$, E. Ros ${ }^{2,1}$, M. A. Pérez-Torres ${ }^{3}$, J. C. Guirado ${ }^{2}$, S. Jiménez-Monferrer ${ }^{2}$, and J. M. Marcaide ${ }^{2}$ \\ 1 Max-Planck-Institut für Radioastronomie, Auf dem Hügel 69, 53121 Bonn, Germany \\ e-mail: imartiv@mpifr.de \\ 2 Departament d'Astronomia i Astrofísica, Universitat de València, 46100 Burjassot, València, Spain \\ 3 Instituto de Astrofísica de Andalucía, CSIC, Apdo. Correos 2004, 08071 Granada, Spain
}

Received 5 February 2010 / Accepted 8 March 2010

\begin{abstract}
Context. Phase-referencing is a standard calibration technique in radio interferometry, particularly suited for the detection of weak sources close to the sensitivity limits of the interferometers. However, effects from a changing atmosphere and inaccuracies in the correlator model may affect the phase-referenced images, and lead to wrong estimates of source flux densities and positions. A systematic observational study of signal decoherence in phase-referencing and its effects in the image plane has not been performed yet.

Aims. We systematically studied how the signal coherence in Very-Long-Baseline-Interferometry (VLBI) observations is affected by a phase-reference calibration at different frequencies and for different calibrator-to-target separations. The results obtained should be of interest for a correct interpretation of many phase-referenced observations with VLBI.

Methods. We observed a set of 13 strong sources (the S5 polar cap sample) at 8.4 and $15 \mathrm{GHz}$ in phase-reference mode with 32 different calibrator/target combinations spanning angular separations between 1.5 and 20.5 degrees. We obtained phase-referenced images and studied how the dynamic range and peak flux-density depend on observing frequency and source separation.

Results. We obtained dynamic ranges and peak flux densities of the phase-referenced images as a function of frequency and separation from the calibrator. We compared our results with models and phenomenological equations previously reported.

Conclusions. The dynamic range of the phase-referenced images is strongly limited by the atmosphere at all frequencies and for all source separations. The limiting dynamic range is inversely proportional to the sine of the calibrator-to-target separation. Not surpriseingly, we also find that the peak flux densities decrease with source separation, relative to those obtained from the selfcalibrated images.
\end{abstract}

Key words. techniques: interferometric - atmospheric effects - instrumentation: interferometers

\section{Introduction}

Phase-referencing is a standard calibration technique in radio interferometry. It allows the detection of a weak source (target source) by using quasi-simultaneous observations of a closeby strong source (calibrator) (see e.g., Ros 2005, and references therein). This technique also allows the user to recover the position of the target source relative to that of the calibrator; a position otherwise lost by the use of closure phases in the imaging. Basically, phase-referencing consists of estimating the antenna-based complex gains with the calibrator fringes, timeinterpolating these gains to the observations of the target, and calibrating the visibilities of the target with the interpolated gains. Therefore, it is assumed that for each antenna the optical paths of the signals from both sources are similar. However, atmospheric turbulences and/or inaccuracies in the correlator geometrical model may introduce errors in the estimates of the optical paths of the signals and severely affect the phasereferencing. These errors can be partially corrected by applying the self-calibration algorithm (see, e.g., Readhead \& Wilkinson 1978) after phase-referencing. However, self-calibration, specially on observations of weak sources, may affect the resulting

\footnotetext{
* Alexander von Humboldt Fellow.
}

images in undesirable ways (see, e.g., Martí-Vidal \& Marcaide 2008).

The correlator model includes contributions from the dry troposphere, the Earth orientation parameters, gain corrections for the sampling, and feed rotation of the alt-azimuthal mounts of the antennae. An imperfect modelling in any of these contributions and the loss of coherence of the radio waves within the time elapsed between consecutive observations of a given source, have an impact on the quality of phase-referencing. Some authors have stated that the loss of signal coherence in phase-referencing is linearly dependent on the separation between the calibrator source and the target source (Beasley \& Conway 1995). However, in a recent publication Martí-Vidal et al. (2010) suggest a phenomenological model different from that of Beasley \& Conway (1995), based on Monte Carlo simulations of atmospheric turbulences.

To empirically establish this dependence, we need to compare phase-referenced images to those obtained from the selfcalibrated visibilities (i.e., the images obtained by applying the optimum phase gains; those that maximize the signal coherence of the target sources). For this purpose, both calibrator and target must be strong enough to generate fringes with a signal-tonoise ratio (SNR) high enough to allow for an accurate estimate of the phase, delay, and rate of the fringe peaks, thus avoiding 
the bias effects related to self-calibration of weak signals (e.g., Martí-Vidal \& Marcaide 2008).

The S5 polar cap sample (a subset of 13 sources from the S5 survey, see Kühr et al. 1981; Eckart et al. 1986) is an ideal set of sources to perform such a study. The spectra of these sources are relatively flat at radio wavelenghts and their flux densities range from hundreds of mJy to several Jy. Therefore it is possible to study the loss of phase coherence as a function of observing frequency and source separation. We observed the S5 polar cap sample at $8.4 \mathrm{GHz}$ and $15 \mathrm{GHz}$. These observations are part of a large astrometry programme (Ros et al. 2001; Pérez-Torres et al. 2004; Martí-Vidal et al. 2008; Jiménez-Monferrer et al. in preparation) and were performed in phase-reference mode with many different calibrator/target combinations. From these observations, we studied the loss of signal coherence in phase-referenced observations by comparing phase-referenced and self-calibrated images for all possible calibrator/target combinations allowed by the observations.

In the next section we describe our observations and the process of data calibration and reduction. In Sect. 3 we report on the results obtained. In Sect. 4 we summarize our conclusions.

\section{Observations and data reduction}

We observed the 13 sources of the S5 polar cap sample with the complete Very Long Baseline Array (VLBA) at $8.4 \mathrm{GHz}$ on 2001 February 3 and at $15 \mathrm{GHz}$ on 2000 June 15. At each epoch, 8 bands of $8 \mathrm{MHz}$ bandwidth each were recorded, obtaining a synthesized bandwidth of $64 \mathrm{MHz}$ in one polarization (LCP). All three epochs consisted of $24 \mathrm{~h}$ of observations. The observations took place in phase-referenced mode with different subsets of three or four radio sources in different duty cycles (see Martí-Vidal et al. 2008 for more details on the observing schedule). The sources of each subset were observed cyclically for about two hours. Each radio source was observed a total of about four hours. Data were cross-correlated at the Array Operation Center of the National Radio Astronomy Observatory (NRAO). Details on the source images at $8.4 \mathrm{GHz}$ and $15 \mathrm{GHz}$ can be found in Ros et al. (2001) and Pérez-Torres et al. (2004), respectively. The results of the high-precision astrometry analysis at $15 \mathrm{GHz}$ can be found in Martí-Vidal et al. (2008). Those at 8.4 GHz will be published elsewhere (Jiménez-Monferrer et al., in preparation).

The data calibration and reduction was performed using ParselTongue (Kettenis et al. 2006), a Python interface to the NRAO Astronomical Image Processing System (AIPS). We generated a script in ParselTongue to automatize the calibration and imaging of the phase-referenced images between all source pairs that were observed within the same duty cycles. A total of 32 source pairs were obtained, covering a range of source separations between 1.5 and 20.5 degrees. We checked the quality of the automated calibration and imaging by re-generating manually phased-referenced images of some pairs of sources and comparing them to the automated images. The results obtained were compatible within $0.1 \sigma$. Below we summarize the process of calibration followed in our analysis.

- Step 1. A correction of the the Earth Orientation Parameters (EOP) as estimated by the United States Naval Observatory (USNO) was applied to the data.

- Step 2. The visibility phases were aligned between the subbands, through the whole $64 \mathrm{MHz}$ band for all sources and times, by fringe-fitting the sub-band delays of a selected scan with high-quality fringes and applying the estimated delays and phases to all visibilities.

- Step 3. A second fringe fitting, using now the delays determined from the whole band, provided new phase corrections for all the observations. The visibility amplitude calibration was performed with the system temperatures and gain curves from each antenna.

- Step 4. The calibrated data were exported into the programme DIFMAP (Shepherd et al. 1995) to obtain images of all sources. The CLEAN algorithm and several iterations of phase and gain self-calibration were applied to each source until high-quality images (with residuals close to the thermal noise) were obtained.

- Step 5. These images were imported back into AIPS for a second fringe fitting, now taking into account the contribution of the source structures in the estimates of the residual delays and phases. The amplitude calibration was also refined by estimating the amplitude gains based on the source structures (one gain solution for all antennae every $10 \mathrm{~min})$.

Once the data were calibrated as described above, we proceeded with the analysis. For each pair of sources (A and B), four sets of data were generated. On the one hand, the self-solutions of A (B) in the time range when this source was observed in the same duty cycles as B (A). On the other hand, the set of visibilities of A (B) phase-referenced to B (A), obviously for the same time range as that of the corresponding self-calibrated data sets. Each of these data sets was used to generate an image using natural weighting of the visibilities (to optimize the sensitivity) and the same CLEAN windows that were used to obtain the images in Step 4 of the data calibration procedure. For the phase-referenced images, the position of the peak flux-density was first estimated and the CLEAN windows were accordingly shifted before CLEANing.

\section{Results and discussion}

\subsection{Dynamic range}

The dynamic ranges of the images obtained as described in the previous section were computed as the peak flux-density per unit beam divided by the root-mean-square (rms) of the image residuals (i.e., after subtracting the CLEAN model). The peak flux densities of the images obtained from the self-calibrated visibilities range between 0.16 and $2.60 \mathrm{Jy}$ at $8.4 \mathrm{GHz}$ and between 0.16 and $1.86 \mathrm{Jy}$ at $15 \mathrm{GHz}$. The typical $\mathrm{rms}$ of these images are $1 \mathrm{mJy}$ beam $^{-1}$ at $8.4 \mathrm{GHz}$ and $2 \mathrm{mJy}^{\mathrm{meam}}{ }^{-1}$ at $15 \mathrm{GHz}$.

A total of 64 phase-referenced images were obtained at each frequency. These are two images for each pair of sources (i.e., image of source A phase-referenced to B and image of source B phase-referenced to A). We then discarded the images with dynamic ranges lower than 10 , because in these cases there is a high chance of confusion of the source with a spurious noise peak. Applying this cutoff to the dynamic ranges, 53 phase-referenced images were left at $8.4 \mathrm{GHz}$ and 31 images at $15 \mathrm{GHz}$. The dynamic ranges of the phase-referenced images are typically a factor $\sim 40$ smaller than those obtained from the self-calibrated visibilities (see Figs. 1 and 2). A first conclusion is that the loss of phase coherence strongly affects the dynamic range of the phase-referenced images, regardless of the calibrator-to-target separation.

A similar conclusion was reported by Martí-Vidal et al. (2010) based on Monte Carlo simultations of phase-referenced 


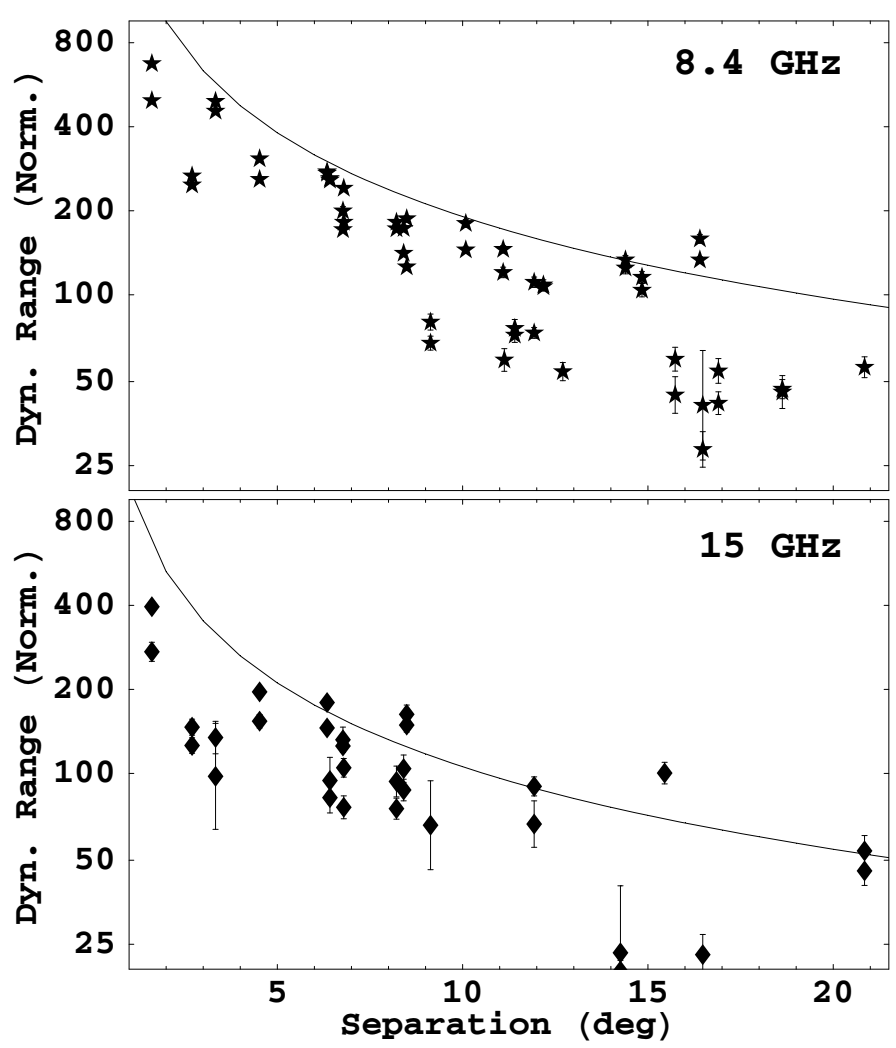

Fig. 1. Dynamic ranges (normalized to an observing time of $10 \mathrm{~h}$ ) of the phase-referenced images as a function of distance to the calibrators. The error bars are proportional to the flux densities of the calibrators. Lines represent a model of the maximum achievable dynamic range (Eq. (2)).

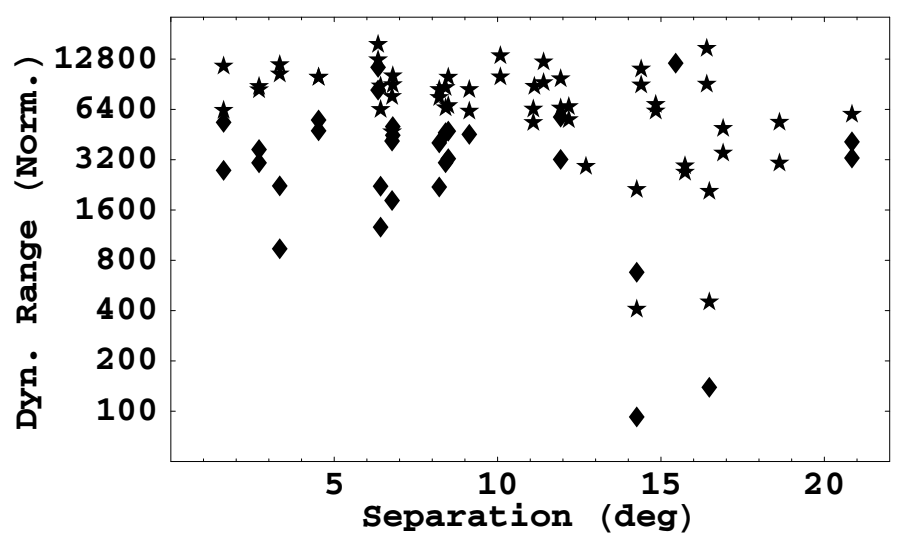

Fig. 2. Dynamic ranges (normalized to an observing time of $10 \mathrm{~h}$ ) of the images obtained from self-calibrated visibilities. Stars are data at $8.4 \mathrm{GHz}$ and diamonds at $15 \mathrm{GHz}$. This figure can be compared to Fig. 1 to see the effect of coherence losses in the phase-referenced images.

observations under a turbulent atmosphere. These authors modelled the dynamic range of a phase-referenced image considering the addition in quadrature of two sources of noise; one due to the thermal noise of the receiving system, $\sigma_{\text {th }}$, and the other due to the atmosphere. $\sigma_{\text {at }}$. The latter was assumed to be equal to a given percentage of the source flux-density, i.e. $\sigma_{\text {at }}=f_{\text {at }} S$. In this expression, $S$ is the flux-density and $f_{\text {at }}$ is a factor that depends on the calibrator-to-target separation, $\theta$, the observing frequency, $v$, and the on-source observing time $\Delta t$. We notice that a similar expression is usually employed in the estimate of the (instrumental) systematic amplitude-calibration errors in VLA and VLBI observations. However, in our case the factor $f_{\text {at }}$ depends on the calibrator-to-target separation and on the total on-source observing time, while the factors for the estimate of systematic amplitude-calibration errors in VLA and VLBI observations only depend on the observing frequency. The dynamic range is thus

$D=\frac{S}{\sqrt{\sigma_{\text {th }}^{2}+f_{\text {at }}^{2} S^{2}}}$.

The dynamic range of a phase-referenced image is limited to a given value, $D_{1}$,

$D_{1}=\frac{1}{f_{\text {at }}}$.

This limit is independent of both $S$ and the sensitivity of the array. It is achieved when the flux-density of the target source is much higher than the thermal noise of the interferometer. The limiting dynamic range only depends on $\theta, v$, and $\Delta t$, and it can be several orders of magnitude smaller than the corresponding dynamic range due to the thermal noise of the receiving system, indicating that the atmosphere strongly limits the sensitivity of the observations. Martí-Vidal et al. (2010) propose a phenomenological model for $f_{\text {at }}$ based on their Monte Carlo simulations. From their Sect. 5.3, it can be derived that

$f_{\text {at }}=K \frac{v}{\sqrt{\Delta t} \sin (\theta)}$

where $K$ is a constant to be determined.

We can compare this phenomenological model to our observations by plotting the dynamic range of all our phase-referenced images as a function of calibrator-to-target separation. The images obtained for each source pair correspond to data with slightly different on-source observing times. Therefore, we corrected (i.e., we normalized) the dynamic ranges of all the images by applying the factor $\sqrt{N_{10} / N_{v}}$, where $N_{v}$ is the number of visibilities for each image and $N_{10}$ is the number of visibilities corresponding to a on-source observing time of 10 hours with the VLBA. We show in Fig. 1 the normalized dynamic ranges and the model resulting from Eq. (3). We find that for $K \sim 12.4 \mathrm{~h}^{0.5} \mathrm{GHz}^{-1}$ the model predicts the limiting dynamic ranges obtained with the VLBA at both frequencies, although the results at $8.4 \mathrm{GHz}$ are of higher quality; the results at $15 \mathrm{GHz}$ are noisier. The dynamic ranges (also normalized to an observing time of 10 hours) obtained from the self-calibrated images are shown in Fig. 2 for a comparison with those obtained from phase-referencing. The error bars in Fig. 1 are set to be proportional to the flux-density of the calibrator. This way the reader has information in a single figure on the quality of the phase-referenced images and the quality of the calibrator visibilities.

\subsection{Peak flux-density}

We show in Fig. 3 the peak flux densities of the phase-referenced images relative to those obtained from the images corresponding to the self-calibrated visibilities as a function of distance to the calibrator. The systematics in the loss of flux-density is clear for the $8.4 \mathrm{GHz}$ data. The flux-density recovered is about $80 \%$ of the real flux-density for separations of $\sim 5$ degrees, and slowly decreases to $50 \%$ for the largest separations. For the 


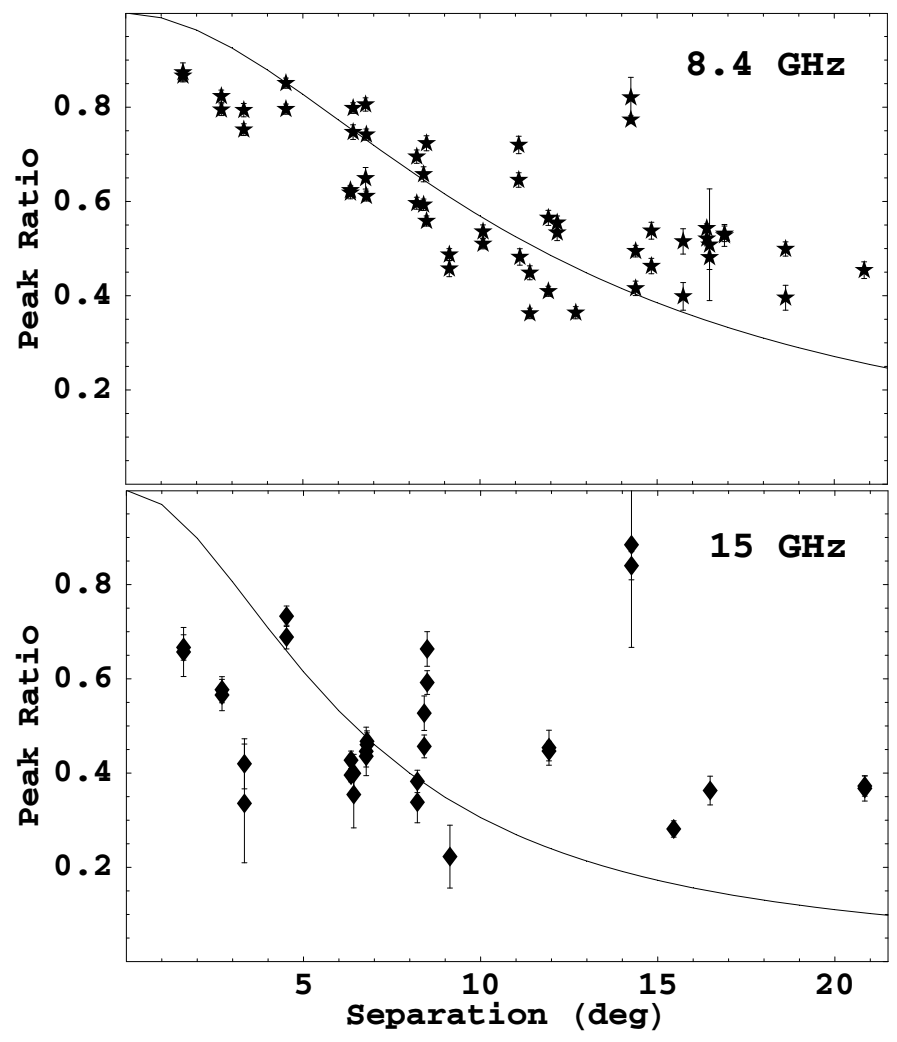

Fig. 3. Peak flux densities of the phase-referenced images normalized to the peak flux densities of the images obtained from the self-calibrated visibilities. Lines represent the model given by Eq. (4).

$15 \mathrm{GHz}$ data, the scatter is larger and no robust conclusion can be obtained. However, a hint of saturation of the peak ratios around $40 \%$ can be appreciated at large source separations if the points corresponding to a separation of 14.84 degrees (for which peak ratios as high as $80 \%$ are obtained) are not considered (these points correspond to sources B1803+784 and B1150+812 phase-referenced to each other).

From the simulations reported in Martí-Vidal et al. (2010), the peak flux-density of a phase-referenced image, $P_{\mathrm{ph}}$, relative to the real peak flux-density of the source, $P_{\mathrm{sf}}$, can be estimated as

$\frac{P_{\mathrm{ph}}}{P_{\mathrm{sf}}}=\frac{1}{1+k_{1} f_{\mathrm{at}}^{k_{2}}}$,

where $k_{1}$ is a constant to be determined. From the Monte Carlo simulations, the exponent $k_{2}$ takes the value $1.87 \pm 0.02$. In Fig. 3 we also show the predictions of the model given by Eq. (4) for $k_{1} \sim 63$. The model roughly predicts the behaviour of the $8.4 \mathrm{GHz}$ data, although for separations larger than $\sim 15 \mathrm{de}-$ grees, the observed peak ratios clearly saturate at $\sim 0.5$, while the model predictions monotonically decrease. A similar conclusion is obtained for the $15 \mathrm{GHz}$ data, where a saturation around 0.4 is appreciated. The saturation in the ratio of flux-density peaks at large calibrator-to-target separations is not modelled using Eq. (4) and could be due to the saturation of the powerspectrum of the tropospheric turbulences at large scales (see, e.g., Thomson et al. 1991), which was not considered in the simulations reported in Martí-Vidal et al. (2010). This saturation of the power spectrum of the turbulences would stabilize the phase difference between target and calibrator for large separations and therefore enhance the signal coherence (and peak flux-density) of the target source.

\section{Conclusions}

We report how phase referencing affects the signal coherence (and the fidelity of the images) in VLBI observations at different frequencies $(8.4 \mathrm{GHz}$ and $15 \mathrm{GHz}$ ) and for different calibratorto-target separations (from 1.5 to 20.5 degrees). We determined the loss of dynamic range and peak flux-density of the phasereferenced images and compared the results with the model predictions given in Martí-Vidal et al. (2010).

The dynamic range of the phase-referenced images is strongly limited by the atmosphere at all frequencies and for all calibrator-to-target separations. The maximum achievable dynamic range using the VLBA is given by Eqs. (2) and (3), with $K \sim 12.4$ (if $v$ is given in $\mathrm{GHz}$ and $\Delta t$ in hours). If the target source is not strong (as is usually the case), the thermal noise of the receiving system cannot be ignored and the dynamic range should be estimated with Eqs. (1) and (3).

The recoverable flux-density (computed as the peak fluxdensity of the phase-referenced image in units of the real peak flux-density of the source) decreases as the separation to the calibrator increases and is given by Eqs. (4) and (3), with $k_{1} \sim 63$ and $k_{2} \sim 1.87$. This model roughly predicts the peak ratios observed at $8.4 \mathrm{GHz}$ for calibrator-to-target separations below $\sim 15$ degrees (the results at $15 \mathrm{GHz}$ are too noisy to reach a robust conclusion). For separations larger than 15 degrees, the observed peak ratios are higher than the model predictions, possibly due to a saturation in the power spectrum of the tropospheric turbulences at large scales, which was not considered in Martí-Vidal et al. (2010).

It is remarkable that for relatively small separations (below 5 degrees), which are typical in many phase-referencing observations, the flux-density loss can be as large as $20 \%$ at $8.4 \mathrm{GHz}$ and $30-40 \%$ at $15 \mathrm{GHz}$ (and even larger at higher frequencies, according to Eq. (4)). It must be also taken into account that the phase-referenced observations here reported were performed under good weather conditions and when the sources were close to their transits at nearly all stations (except Mauna Kea and St. Croix, see Martí-Vidal et al. 2008, for details on the observing schedule). Therefore even larger flux-density losses and lower dynamic ranges may be obtained when the observing conditions are far from optimal.

However, we must also notice that the typical calibrator-tocalibrator cycle times in our observations were about 120-180 s. For observations at $8.4 \mathrm{GHz}$, these cycle times are of the order of (and slightly shorter than) the coherence time of the signal (which takes typical values of 180-240 s in VLBI observations). Therefore it is not expected that a changing atmosphere may have introduced strong effects in the coherence of the phasereferenced visibilities at $8.4 \mathrm{GHz}$. At $15 \mathrm{GHz}$, the shorter coherence time (which takes typical values of 100-140 s in VLBI observations) might be short enough to imply an additional phase degradation in the phase-referenced visibilities due to a changing atmosphere. Nevertheless, this issue is unlikely to significantly affect the results of our analysis, because the time evolution of the self-solutions of the antenna-based phase gains at $15 \mathrm{GHz}$ are smooth, which indicates that the weather conditions were good enough to allow for a well-behaved phase connection between contiguous scans of each source.

Acknowledgements. I.M.V. is a fellow of the Alexander von Humboldt Foundation in Germany. The Very Long Baseline Array is operated by the 
I. Martí-Vidal et al.: Coherence loss in phase-referenced VLBI observations

USA National Radio Astronomy Observatory, which is a facility of the National Science Foundation operated under cooperative agreement by Associated Universities, Inc. Partial support was obtained by Generalitat Valenciana (Prometeo 2009 P104) and from Spanish grant AYA 2005-08561-C03-02. MAPT acknowledges support by the MEC through grant AYA 2006-14986C02-01, and by the Consejería de Innovación, Ciencia y Empresa of Junta de Andalucía through grants FQM-1747 and TIC-126.

\section{References}

Beasley, A. J., \& Conway, J. E. 1995, in Very Long Baseline Interferometry and the VLBA, ed. J. A. Zensus, P. J. Diamond, \& P. J. Napier (San Francisco: ASP), ASP Conf. Ser., 82, 327

Eckart, A., Witzel, A., Biermann, P., et al. 1986, A\&A, 168, 17

Martí-Vidal I., \& Marcaide, J. M. 2008, A\&A 480, 289
Martí-Vidal, I., Marcaide, J. M., Guirado, J. C., Pérez-Torres, M. A., \& Ros, E. 2008, A\&A 478, 267

Martí-Vidal, I., Guirado, J. C., Marcaide, J.M., \& Jiménez-Monferrer, S. 2010, A\&A, accepted [arXiv: 1004 .1624]

Pérez-Torres, M. A., Marcaide, J. M., Guirado, J. C., \& Ros, E. 2004, A\&A, 428, 847

Kettenis, M., van Langevelde, H. J., Reynolds, C., \& Cotton, B. 2006, ASPC, 351,497

Kühr, H., Witzel, A., Pauliny-Toth, I. I. K., et al. 1981, A\&AS, 45, 367

Readhead, A. C. S., \& Wilkinson, P. N. 1978, ApJ, 223, 25

Ros, E. 2005, in Future Directions in High Resolution Astronomy: The 10th Anniversary of the VLBA, ASP Conference Proceedings, ed. J. Romney, \& M. Reid (San Francisco: ASP), ASP Conf. Ser., 340, 482

Ros, E., Marcaide, J. M., Guirado, J. C., \& Pérez-Torres, M. A. 2001, A\&A, 348, 381

Shepherd, M. C., Pearson, T. J., \& Taylor, G. B. 1995, BAAS, 26, 987

Thomson, A. R., Moran, J. M., \& Swenson, G. W. 1991, Interferometry and Synthesis in Radio Astronomy (Florida: Krieger Publ. Corp.) 